INPLASY

PROTOCOL

To cite: Xiang et al. Machine learning progress in lower limb running biomechanics with wearable technology. Inplasy protocol 202210083. doi: 10.37766/inplasy2022.1.0083

Received: 16 January 2022

Published: 16 January 2022

Corresponding author:

Liangliang Xiang

xiangliang03@hotmail.com

Author Affiliation:

University of Auckland

Support: None.

Review Stage at time of this submission: Data analysis Completed but not published.

Conflicts of interest:

None declared.

\section{Machine learning progress in lower limb running biomechanics with wearable technology}

Xiang, L1; Wang, A2; Gu, Y3; Shim, V4; Fernandez, J5.

Review question / Objective: The purpose of this initial study is to conduct a systematic review regarding machine learning and deep learning approaches used in running biomechanics and was limited to the wearable sensors placed in lower limbs.

Condition being studied: With the emergence of wearable technology and machine learning approaches, gait monitoring in real-time is attracting interest from the sports biomechanics community. This study presents a systematic review of machine learning approaches in running biomechanics using wearable sensors. Wearable sensors monitoring.

INPLASY registration number: This protocol was registered with the International Platform of Registered Systematic Review and Meta-Analysis Protocols (INPLASY) on 16 January 2022 and was last updated on 16 January 2022 (registration number INPLASY202210083).

\section{INTRODUCTION}

Review question / Objective: The purpose of this initial study is to conduct a systematic review regarding machine learning and deep learning approaches used in running biomechanics and was limited to the wearable sensors placed in lower limbs.

Condition being studied: With the emergence of wearable technology and machine learning approaches, gait monitoring in real-time is attracting interest from the sports biomechanics community. 
This study presents a systematic review of machine learning approaches in running biomechanics using wearable sensors. Wearable sensors monitoring.

\section{METHODS}

Search strategy: This systematic review followed the PRISMA (Preferred Reporting Items for Systematic Reviews and MetaAnalyses) recommendations. Electronic databases were retrieved in PubMed, Web of Science, SPORTDiscus, Scopus, IEEE $X p l o r e$, and ScienceDirect by one reviewer (L.X.) to identify original research articles published up to 15th January 2021 (from 2000-). After an initial search, relative article information was input to Rayyan QCRI for duplicate removal, study screening, and identification. One study was added from another source to review flow for further paper screening. NonEnglish articles and conference proceedings, and dissertations were excluded. The retrieve strategy and limit conditions are shown in Table 1. Four different categories were used to identify relevant studies: wearable inertial sensor, machine learning and deep learning, lower limb, and running. By using Boolean operation, the retrieved studies at least contain one keyword in the full field. Electronic databases were retrieved in PubMed, Web of Science, SPORTDiscus, Scopus, IEEE Xplore, and ScienceDirect by one reviewer to identify original research articles published up to 15th January 2021 (from 2000-). After an initial search, relative article information was input to Rayyan QCRI for duplicate removal, study screening, and identification.

\section{Participant or population: Healthy runners.}

Intervention: Our research identified studies that utilized machine learning and deep learning approaches and wearable inertial sensors to assess running biomechanics.

\section{Comparator: Not applicable.}

Study designs to be included: All studies will be either randomised controlled trials
(RCTs), non-randomised controlled trials (nRCTs) or non-randomised non-controlled trials (nRnCTs).

Eligibility criteria: Articles were selected based on the PICO principle (participants, intervention, comparisons, and outcomes). Our research identified studies that utilized machine learning and deep learning approaches and wearable inertial sensors to assess running biomechanics. For the included studies, wearable sensors must be placed in the participants' lower limbs. Only inertial sensors were selected in this review, including accelerometer, gyroscope, and magnetometer. Studies were removed if the statistical regression model rather than the machine learning approach was employed for the prediction. Energy expenditure and sprint performance assessment studies were not considered in this study. Studies using machine learning and wearable sensors only evaluating walking gait and human activity recognition were excluded.

Information sources: Electronic databases were retrieved in PubMed, Web of Science, SPORTDiscus, Scopus, IEEE Xplore, and ScienceDirect.

Main outcome(s): Twenty-four articles met the eligibility criteria after article screening was included in this systematic review. The range of quality scores of the included studies are from 0.78 to 1.00 , with $40 \%$ of articles recruiting participant numbers between 20 to 50 . The number of inertial measurement unit (IMU) placed on the lower limbs varied from 1 to 5 , mainly in the pelvis, thigh, distal tibia, and foot. Deep learning algorithms occupied $57 \%$ of total machine learning approaches. Convolutional neural networks (CNN) was the most frequently used deep learning algorithm. However, test or validation of the machine learning models were lacking and needed for future studies. The deep learning model combining multiple CNN and recurrent neural networks (RNN) was observed to extract different running features from the wearable sensors and presents a growing trend in running biomechanics. 
Quality assessment / Risk of bias analysis: The methodological quality of the included studies was assessed by the modified QualSyst Assessment Tool for quantitative studies. Ten items were identified in this scale for aspects of the research question, study design, statistical analysis, sample size, and results reporting. Each item was scored as 'yes' $=2$, 'partial' $=1$, or ' $n o '=0$ in view of the degree of the specific criteria that were met. The risk of bias was initially evaluated by one investigator (L.X.), rechecked by two authors (A.W., J.F.). Each study's score was calculated by summing ten items' scores and dividing by the total score.

Strategy of data synthesis: Not applicable. This review will do no data synthesis.

Subgroup analysis: Not applicable.

Sensitivity analysis: Not applicable.

Language: English.

Country(ies) involved: New Zealand, China.

Keywords: Gait; Wearable sensor; Machine learning; Deep learning; Running; Lower limb.

Contributions of each author:

Author 1 - Liangliang Xiang.

Author 2 - Wang, A.

Author 3 - Gu, Y.

Author 4 - Shim, V.

Author 5 - Fernandez, J. 